\title{
Use of the ICD-10 vision codes to study ocular conditions in Medicare beneficiaries with stroke
}

Kimberly P. Hreha ${ }^{1 *} \mathbb{D}$, Steve R. Fisher ${ }^{2}$, Timothy A. Reistetter ${ }^{3}$, Kenneth Ottenbacher ${ }^{1}$, Allen Haas $^{4}$, Chih-Ying Li ${ }^{5}$, Joshua R. Ehrlich ${ }^{6}$, Diane B. Whitaker ${ }^{7}$ and Heather E. Whitson ${ }^{8,9,10}$

\begin{abstract}
Background: Ocular conditions are common following stroke and frequently occur in combination with preexisting ophthalmologic disease. The Medicare International Statistical Classification of Diseases and Related Health Problems (ICD-10) coding system for identifying vision related health conditions provides a much higher level of detail for coding these complex scenarios than the previous ICD-9 system. While this new coding system has advantages for clinical care and billing, the degree to which providers and researchers are utilizing the expanded code structure is unknown. The purpose of this study was to describe the use of ICD-10 vision codes in a large cohort of stroke survivors.
\end{abstract}

Methods: Retrospective cohort design to study national 100\% Medicare claims files from 2015 through 2017. Descriptive data analyses were conducted using all available ICD-10 vision codes for beneficiaries who had an acute care stay because of a new stroke. The outcome of interest was $\geq 1$ ICD-10 visual code recorded in the claims chart.

Results: The cohort ( $n=269,314)$ was mostly female (57.1\%) with ischemic stroke (87.8\%). Approximately 15\% were coded as having one or more ocular condition. Unspecified glaucoma was the most frequently used code among men $(2.83 \%)$, those over $85+(4.80 \%)$ and black beneficiaries (4.12\%). Multiple vision codes were used in few patients (0.6\%). Less than $3 \%$ of those in the oldest group (85+ years) had two or more vision codes in their claims.

Conclusions: Ocular comorbidity was present in a portion of this cohort of stroke survivors, however the vision codes used to describe impairments in this population were few and lacked specificity. Future studies should compare ophthalmic examination results with billing codes to characterize the type and frequency of ocular comorbidity. It important to understand how the use of ICD-10 vision codes impacts clinical decision making, recovery, and outcomes.

Keywords: Vision, Impairment, Ocular conditions, Stroke, Comorbidity, Medical coding

\footnotetext{
* Correspondence: khreha318@gmail.com

'Division of Rehabilitation Sciences, University of Texas Medical Branch, School of Health Professions, 301 University Blvd., Galveston, TX 77555, USA

Full list of author information is available at the end of the article
} 


\section{Background}

Stroke is the leading cause of disability in the United States (U.S.) and worldwide [1]. One major disability resulting from stroke is ocular conditions and vision impairment [1]. For acute stroke survivors, the prevalence of vision impairments has been reported to range from 65 to $92 \%[2-4]$ and the incidence of new visual impairments, at stroke onset was $59.6 \%$ [3]. Approximately $60 \%$ of people with chronic stroke report vision deficits including ocular deficits [5]. Stroke or neurologically-related vision or eye problems typically include conditions such as visual field loss, ptosis, strabismus, or oculomotor impairments [4]. However stroke survivors may also have an elevated risk of other types of vision impairments that share common risk factors with stroke, such as diabetic retinopathy, since diabetes is a common risk factor for stroke and vision impairment [6]. Additionally, stroke survivors may have comorbid ophthalmologic diseases, particularly conditions such as glaucoma, age-related macular degeneration, and cataracts, that are common among older adults [3, 7].

Healthcare providers use hospital medical coding to classify diagnoses and reasons for medical visits. The Medicare International Statistical Classification of Diseases and Related Health Problems (ICD-10) codes, which replaced ICD-9 codes on October 1, 2015, is the current coding system used in the U.S. The ICD-10 system brought changes which included: rearrangement of parts of the code book, alphanumeric characters used for all codes, modernization and harmonization of the terminology, the use of new codes combining diagnoses and symptoms, as well as a significant increase in the specificity of the reporting. Differences between ICD-9 and ICD-10 are highlighted by number alone-- there are over 71,000 diagnostic codes in the ICD-10 versus approximately 13,000 ICD-9 codes [8]. The level of detail present in the ICD-10 codes requires much greater specificity in medical documentation and code selection.

While the granularity of the new coding system has advantages for clinical care, the added complexity may create challenges for population health research and even hospital billing providers. For example, it may be more time intensive for providers to search through all 71,000 codes to determine the most appropriate code to use for every patient admitted for medical services. The objective of this study was to determine the patterns of use of ICD-10 codes to identify vision or eye problems in a national stroke cohort. We hypothesized that the range of codes used in a stroke cohort would be limited and biased toward neuro-ophthalmic diagnoses, because of the inciting neurological event. We further conjectured that stroke survivors would be likely to have multiple eye and vision codes, including non-neurologic codes, since age and certain medical conditions are common risk factors for eye disease and stroke.

\section{Methods}

We employed a retrospective cohort design and nationwide 100\% Medicare claims data between October 1, 2015 and December 31, 2017. This study use data from a parent study that is examining rehabilitation services and was approved by the Institutional Review Board at the University of Texas Medical Branch. This organization also holds a data usage agreement with the Centers for Medicare and Medicaid Services.

\section{Study sample}

The population of interest was participants who had an acute care stay because of a primary diagnosis of stroke based on the Rehabilitation Impairment Category codes 01.1, 01.2, 01.3, 01.4, 01.9 who also had an ICD-10 vision/ eye code recorded at least once in their claims file $(n=1$, 106,141). Based on our exclusion criteria, we removed beneficiaries without a post-acute care claim within 30 days of hospital discharge, to be able to determine whether the stroke codes used are incident or prevalent diagnoses $(n=712,499)$. Then we removed those whose hospital admission or post-acute care discharge was outside our study window and those under age $18(n=63$, 433). Finally, we removed those without continuous Medicare coverage for 90 days before the hospital admission and 31 days after post-acute care discharge, which allow us to track patients continuously $(n=55,888)$. See Fig. 1 for a diagram of our study sample selection.

\section{Study variables \\ Ocular conditions}

The 203 main vision and eye related ICD-10 main codes were used to determine any eye disorder or vision impairment present in the patient's medical record (www.findacode.com). For example, the main code for glaucoma is H40.X. Under the main code are sub-codes. H40.0 through H40.8 are sub-codes for glaucoma, which include information such as type of glaucoma and which eye is affected. These sub-codes provide very detailed information, such as if the glaucoma is secondary to another ocular disorder and the stage of the disease.

\section{Demographic and clinical factors}

Patient characteristics included: 1) age at admission (categorized into the following groups: 18-49, 50-64, 65$74,75-84$, and $85+$ years); 2 ) race/ethnicity (non-Hispanic white, non-Hispanic black, Hispanic, other); 3) type of stroke (hemorrhagic, ischemic); 4) gender (male, female); 5) Medicaid eligibility (yes, no), used as a proxy for socioeconomic status [9]; and 6) the comorbidities listed in the Elixhauser Comorbidity Index [10]. Clinical factors included: 1 ) length of stay in acute care (1-2, 3, 4, 5, 6-7, 8+ days); 2$)$ post-acute care facility (home health, inpatient rehabilitation, long term acute care, or skilled nursing); and 3) stays 


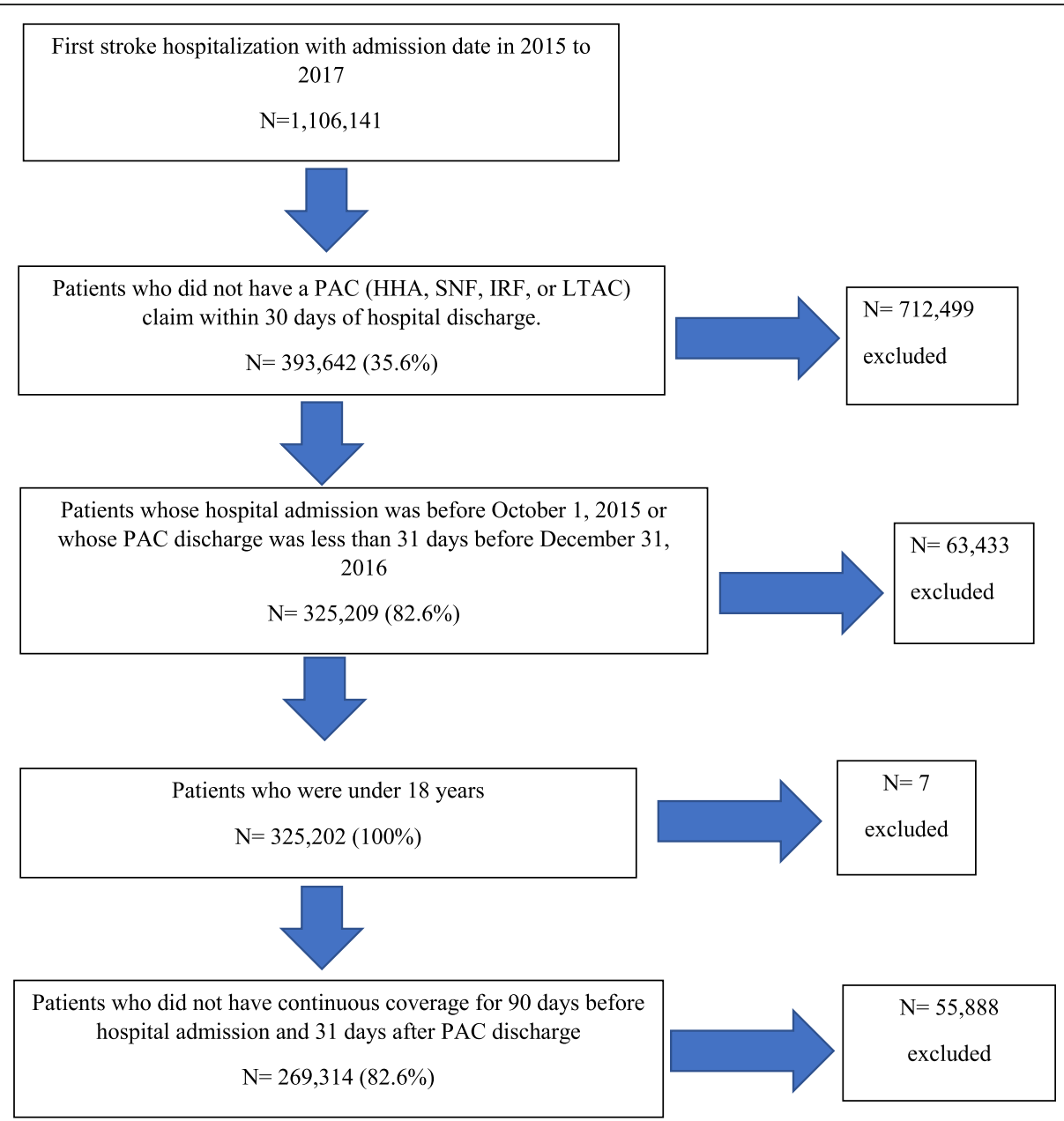

Fig. 1 Consort Diagram of cohort selection. Note: Values in parentheses are the percentage of the previous step remaining; PAC: Post Acute Care; HHA: Home Health Agency; SNF: Skilled Nursing Facility; IRF: Inpatient Rehabilitation Facility; LTAC: Long Term Assistive Care

in the intensive care unit/coronary care unit (yes, no), used as a proxy for stroke severity [11].

\section{Data analysis}

We provided counts and percentages of the sample demographic variables. The codes that were identified as present in the medical chart were codes billed during the post-acute care stays. The five most common eye and vision codes were identified for the entire cohort; we then calculated the prevalence of these codes by demographic and clinical variables. We also calculated the most common eye and vision codes for individuals with each of the 31 medical conditions in the Elixhauser Comorbidity Index [10]. Lastly, frequencies and percentages of the most common codes were stratified by age group. Data management was completed before analysis because it required merging of multiple datasets. The analyses were performed with SAS statistical software Version 9.4 (SAS, Inc., Cary, NC).

\section{Results}

Our initial dataset had 1,106,141 beneficiaries who both sustained a new stroke and had an ocular condition. The final cohort, after application of selection criteria, included 269,314 individuals (Fig. 1). Table 1 presents the characteristics of the study sample. More than half were female $(57.1 \%)$ with a higher prevalence of ischemic stroke compared to hemorrhagic $(87.8 \%$ versus $12.2 \%$, respectively). The most common racial/ethnic group was non-Hispanic white $(76.2 \%)$ and the most common age group was $75-84$ years (33.4\%). In all, $14.8 \%$ of Medicare beneficiaries with stroke were identified as having at least one eye or vision diagnosis upon admission to acute care. Table 1 also describes the percentage with eye and vision codes for each demographic variable. There were roughly an equal percentage of men and women with these codes. Approximately $23 \%$ discharged to inpatient rehabilitation had one or more eye or vision diagnosis. 
Table 1 Cohort Demographics

\begin{tabular}{|c|c|c|}
\hline Demographic & N (\%) & Percentage with Vision Code $(95 \% \mathrm{Cl})$ \\
\hline Overall & 269,314 & $14.8 \%(14.7,14.9)$ \\
\hline \multicolumn{3}{|l|}{ Gender } \\
\hline Male & $115,570(42.9 \%)$ & $14.9 \%(14.7,15.1)$ \\
\hline Female & $153,744(57.1 \%)$ & $14.7 \%(14.6,14.9)$ \\
\hline \multicolumn{3}{|l|}{ Age } \\
\hline $18-49$ & $2997(1.1 \%)$ & $16.5 \%(15.2,17.9)$ \\
\hline $50-64$ & $19,716(7.3 \%)$ & $14.5 \%(14.0,15.0)$ \\
\hline $65-74$ & $71,697(26.6 \%)$ & $14.2 \%(14.0,14.5)$ \\
\hline $75-84$ & $90,078(33.4 \%)$ & $14.2 \%(14.0,14.4)$ \\
\hline $85+$ & $84,826(31.5 \%)$ & $16.0 \%(15.7,16.2)$ \\
\hline \multicolumn{3}{|l|}{ Race/Ethnicity } \\
\hline Non-Hispanic White & $205,306(76.2 \%)$ & $15.0 \%(14.9,15.2)$ \\
\hline Non-Hispanic Black & $37,832(14.0 \%)$ & $14.1 \%(13.7,14.4)$ \\
\hline Hispanic & $15,239(5.7 \%)$ & $14.2 \%(13.7,14.8)$ \\
\hline Other & $10,937(4.1 \%)$ & $13.9 \%(13.2,14.5)$ \\
\hline \multicolumn{3}{|l|}{ Original Entitlement } \\
\hline Age & $216,115(80.2 \%)$ & $15.0 \%(14.8,15.1)$ \\
\hline Disability & $50,591(18.8 \%)$ & $14.0 \%(13.7,14.3)$ \\
\hline End Stage Renal Disease (ESRD) & $1255(0.5 \%)$ & $19.7 \%(17.5,21.9)$ \\
\hline Disability and ESRD & $1353(0.5 \%)$ & $19.6 \%(17.5,21.7)$ \\
\hline \multicolumn{3}{|l|}{ Dual Medicare/Medicaid Eligible } \\
\hline No & $194,910(72.4 \%)$ & $15.6 \%(15.4,15.7)$ \\
\hline Yes & $74,404(27.6 \%)$ & $12.8 \%(12.6,13.1)$ \\
\hline \multicolumn{3}{|l|}{ Intensive Care Unit days } \\
\hline 0 & $119,053(44.2 \%)$ & $14.8 \%(14.6,15.0)$ \\
\hline $1-2$ & $53,501(19.9 \%)$ & $15.2 \%(14.9,15.5)$ \\
\hline $3-4$ & $52,682(19.6 \%)$ & $15.4 \%(15.1,15.7)$ \\
\hline $5+$ & $44,078(16.4 \%)$ & $13.8 \%(13.5,14.1)$ \\
\hline \multicolumn{3}{|l|}{ Length of Acute Stay } \\
\hline $1-2$ & $55,391(20.6 \%)$ & $15.8 \%(15.5,16.1)$ \\
\hline 3 & $64,601(24.0 \%)$ & $15.2 \%(14.9,15.5)$ \\
\hline 4 & $44,591(16.6 \%)$ & $15.5 \%(15.2,15.8)$ \\
\hline 5 & $30,335(11.3 \%)$ & $15.1 \%(14.7,15.5)$ \\
\hline $6-7$ & $34,873(12.9 \%)$ & $14.6 \%(14.2,15.0)$ \\
\hline $8+$ & $39,523(14.7 \%)$ & $12.0 \%(11.6,12.3)$ \\
\hline \multicolumn{3}{|l|}{ Post-Acute Care Type } \\
\hline Home Health Agency & $56,954(21.1 \%)$ & $10.5 \%(10.3,10.8)$ \\
\hline Inpatient Rehabilitation Facility & $101,278(37.6 \%)$ & $22.8 \%(22.5,23.0)$ \\
\hline Long Term Acute Care & $2689(1.0 \%)$ & $10.0 \%(8.9,11.1)$ \\
\hline Skilled Nursing Facility & $108,393(40.2 \%)$ & $9.7 \%(9.6,9.9)$ \\
\hline \multicolumn{3}{|l|}{ Stroke Type } \\
\hline Hemorrhagic & $32,747(12.2 \%)$ & $14.6 \%(14.2,14.9)$ \\
\hline Ischemic & $236,546(87.8 \%)$ & $14.9 \%(14.7,15.0)$ \\
\hline
\end{tabular}


Table 2 presents the most used ICD-10 vision codes by demographics, which were: unspecified glaucoma, unspecified age-related macular degeneration, diplopia, other visual disturbance, and homonymous visual field defect. Unspecified glaucoma was the code most used in the group of $85+$ years $(4.8 \%)$, females $(3.4 \%)$, and non-

Table 2 Most Common ICD-10 Vision Codes by Demographics

\begin{tabular}{|c|c|c|c|c|c|}
\hline Demographic & $\begin{array}{l}\text { H409 (unspecified } \\
\text { glaucoma) }\end{array}$ & $\begin{array}{l}\text { H3530 (unspecified } \\
\text { macular degeneration) }\end{array}$ & $\begin{array}{l}\text { H532 } \\
\text { (diplopia) }\end{array}$ & $\begin{array}{l}\text { H538 (other visual } \\
\text { disturbances) }\end{array}$ & $\begin{array}{l}\text { H53462 } \\
\text { (homonymous } \\
\text { bilateral field } \\
\text { cut, left side) } \\
\end{array}$ \\
\hline \multicolumn{6}{|l|}{ Gender } \\
\hline Male & $3272(2.83 \%)$ & 1409 (1.22\%) & $1904(1.65 \%)$ & $1269(1.10 \%)$ & $1020(0.88 \%)$ \\
\hline Female & $5176(3.37 \%)$ & $3380(2.20 \%)$ & $1797(1.17 \%)$ & $1470(0.96 \%)$ & $1219(0.79 \%)$ \\
\hline \multicolumn{6}{|l|}{ Age } \\
\hline $18-49$ & $26(0.87 \%)$ & * & $53(1.77 \%)$ & $67(2.24 \%)$ & $20(0.67 \%)$ \\
\hline $50-64$ & $239(1.21 \%)$ & $41(0.21 \%)$ & $354(1.80 \%)$ & $289(1.47 \%)$ & $163(0.83 \%)$ \\
\hline $65-74$ & $1353(1.89 \%)$ & $307(0.43 \%)$ & $1490(2.08 \%)$ & 957 (1.33\%) & $734(1.02 \%)$ \\
\hline $75-84$ & $2762(3.07 \%)$ & $1326(1.47 \%)$ & $1222(1.36 \%)$ & $865(0.96 \%)$ & $775(0.86 \%)$ \\
\hline $85+$ & $4068(4.80 \%)$ & $3114(3.67 \%)$ & $582(0.69 \%)$ & $561(0.66 \%)$ & 547 (0.64\%) \\
\hline \multicolumn{6}{|l|}{ Race/Ethnicity } \\
\hline Non-Hispanic White & 6135 (2.99\%) & $4555(2.22 \%)$ & $2946(1.43 \%)$ & $2058(1.00 \%)$ & $1847(0.90 \%)$ \\
\hline Non-Hispanic Black & $1559(4.12 \%)$ & $78(0.21 \%)$ & $422(1.12 \%)$ & $396(1.05 \%)$ & 207 (0.55\%) \\
\hline Hispanic & $417(2.74 \%)$ & $75(0.49 \%)$ & $188(1.23 \%)$ & 154 (1.01\%) & 99 (0.65\%) \\
\hline Other & 337 (3.08\%) & $81(0.74 \%)$ & $145(1.33 \%)$ & $131(1.20 \%)$ & $86(0.79 \%)$ \\
\hline \multicolumn{6}{|l|}{ Reason why on Medicare } \\
\hline Old age & $7399(3.42 \%)$ & $4471(2.07 \%)$ & $2893(1.34 \%)$ & $2108(0.98 \%)$ & $1848(0.86 \%)$ \\
\hline Disability Insurance & 992 (1.96\%) & $306(0.60 \%)$ & $781(1.54 \%)$ & $600(1.19 \%)$ & $387(0.76 \%)$ \\
\hline End Stage Renal Disease (ESRD) & $26(2.07 \%)$ & * & $11(0.88 \%)$ & $16(1.27 \%)$ & * \\
\hline Disability and ESRD & $31(2.29 \%)$ & * & $16(1.18 \%)$ & $15(1.11 \%)$ & * \\
\hline \multicolumn{6}{|l|}{ Dual Medicare/Medicaid Eligible } \\
\hline No & $6501(3.34 \%)$ & $4055(2.08 \%)$ & $3031(1.56 \%)$ & $2125(1.09 \%)$ & $1781(0.91 \%)$ \\
\hline Yes & $1947(2.62 \%)$ & $734(0.99 \%)$ & $670(0.90 \%)$ & $614(0.83 \%)$ & $458(0.62 \%)$ \\
\hline \multicolumn{6}{|l|}{ Intensive Care Unit days } \\
\hline 0 & $3664(3.08 \%)$ & $2337(1.96 \%)$ & $1639(1.38 \%)$ & 1199 (1.01\%) & $899(0.76 \%)$ \\
\hline $1-2$ & $1692(3.16 \%)$ & $1019(1.90 \%)$ & $812(1.52 \%)$ & $576(1.08 \%)$ & $490(0.92 \%)$ \\
\hline $3-4$ & $1736(3.30 \%)$ & 917 (1.74\%) & $731(1.39 \%)$ & $581(1.10 \%)$ & $473(0.90 \%)$ \\
\hline $5+$ & $1356(3.08 \%)$ & $516(1.17 \%)$ & $519(1.18 \%)$ & $383(0.87 \%)$ & $377(0.86 \%)$ \\
\hline \multicolumn{6}{|l|}{ Length of Acute Stay } \\
\hline $1-2$ & $1676(3.03 \%)$ & $1173(2.12 \%)$ & $980(1.77 \%)$ & $715(1.29 \%)$ & $484(0.87 \%)$ \\
\hline 3 & $2155(3.34 \%)$ & $1315(2.04 \%)$ & $917(1.42 \%)$ & $663(1.03 \%)$ & $493(0.76 \%)$ \\
\hline 4 & $1503(3.37 \%)$ & $872(1.96 \%)$ & $632(1.42 \%)$ & $493(1.11 \%)$ & $391(0.88 \%)$ \\
\hline 5 & 967 (3.19\%) & $541(1.78 \%)$ & $398(1.31 \%)$ & $269(0.89 \%)$ & $269(0.89 \%)$ \\
\hline $6-7$ & 1077 (3.09\%) & $536(1.54 \%)$ & $458(1.31 \%)$ & $327(0.94 \%)$ & $307(0.88 \%)$ \\
\hline $8+$ & $1070(2.71 \%)$ & $352(0.89 \%)$ & $316(0.80 \%)$ & $272(0.69 \%)$ & $295(0.75 \%)$ \\
\hline \multicolumn{6}{|l|}{ Post-Acute Care Type } \\
\hline Home Health & $966(1.70 \%)$ & 849 (1.49\%) & $466(0.82 \%)$ & $429(0.75 \%)$ & $255(0.45 \%)$ \\
\hline Inpatient Rehabilitation Facility & $4258(4.20 \%)$ & $2189(2.16 \%)$ & $2849(2.81 \%)$ & $2136(2.11 \%)$ & $1752(1.73 \%)$ \\
\hline Long Term Acute Care & $58(2.16 \%)$ & $15(0.56 \%)$ & $15(0.56 \%)$ & $16(0.60 \%)$ & $12(0.45 \%)$ \\
\hline Skilled Nursing Facility & $3166(2.92 \%)$ & $1736(1.60 \%)$ & $371(0.34 \%)$ & $158(0.15 \%)$ & $220(0.20 \%)$ \\
\hline
\end{tabular}


Hispanic blacks (4.1\%). Glaucoma was also the most common condition identified in the inpatient rehabilitation group (4.2\%). 192 of the 203 vision codes were never used in this cohort. Five of the codes were used with more than $1 \%$ of individuals in this dataset.

The number of codes per patient by age group is shown in Table 3. One code (glaucoma) was found most frequently in the $85+$ group (12.8\%). Two as well as three or more codes were used in all groups, but overall the application of multiple eye and vision codes was infrequent. For example, $2.6 \%$ of those in the oldest group ( $85+$ years) had 2 eye or vision codes noted in their claims.

Table 4 shows which eye and vision codes were used among stroke survivors with the top 31 Elixhauser comorbidities. Glaucoma is also the most common code among people living with chronic heart failure, valvular disease, anemia, and others. In addition, $21.5 \%$ of individuals with any vision code had diabetes, $18 \%$ depression, and $15 \%$ psychoses.

\section{Discussion}

In this nationally representative sample of $100 \%$ Medicare claims files, the application of eye and vision billing codes in an acute stroke population varied by patient demographics, but the frequency of coding for ocular conditions was less than expected. Overall, only a small number of codes were used to describe the ocular conditions in this cohort, despite the very large number of codes now available in ICD-10. It is possible that these codes represent the only ocular conditions that were present in this cohort. However other studies report that older adults have general multimorbidity, which puts them at risk for ocular conditions [12], as well as a high prevalence of ophthalmologic comorbidity [13].

It is noteworthy that the majority of the patients had just one eye/vision code. About $2 \%$ of the sample had two vision impairment codes and less than $1 \%$ had three or more codes. Based on other reports of high prevalence of ocular conditions in stroke $(60 \%)$, we speculate that the number of individuals with multiple vision impairments might be under reported for stroke survivors under Medicare coverage, due to the lack of mandated and systematic assessment procedures [14].

The most frequently used single vision billing code was glaucoma. Specifically, the glaucoma codes were most prevalent among non-Hispanic Blacks, which is consistent with prior research reports of non-Hispanic Blacks have higher prevalence of glaucoma $[15,16]$. Glaucoma was also more common among females and older individuals in our study, as expected for an ocular disease that is strongly age-related. We hypothesized that age-related eye conditions would be present but not at a rate considerably higher than in the general population since this was a post-acute care cohort without ophthalmology clinic data. Thus, this finding was surprising. One possibility is that these codes were taken from past medical histories which could include previous ophthalmology records and specific terminology. This may also have been the case if certain medications (e.g. eye drops for glaucoma) were continued during the inpatient hospital stay. If a diagnosis is not evident but a patient is taking a specific medication at the time of admission, the medical team may investigate further in order to ensure continuation of the person's outpatient eye care.

Currently, only two acute-care based studies have reported the prevalence and frequency of multiple vision impairments in stroke survivors [3, 4]. These were reports that used prospective clinical data and were not restricted to Medicare beneficiaries because they were based in the United Kingdom [3, 4]. Unlike this study's findings ( $15 \%$ of the total group had ocular conditions), the researchers found that approximately $72.8 \%$ of individuals admitted for a stroke had abnormal findings on their ophthalmologic assessments [3]. And because the study was prospective, they were able to report on the timing of the vision assessment including that $13 \%$ had visual problems that pre-existed the stroke, $27 \%$ had both new stroke visual problems and pre-existing vision and ocular impairments and 32\% had new onset strokerelated visual conditions [3]. Similarities were found between this study and ours. For example, our study found that the $85+$ group had the highest percentage (2.6\%) of having two vision codes documented among the group. Rowe et al. reported that the highest percentage of documented visual problems were found among the 80-90- year olds for impaired central vision (18.5\%), visual field loss (8.7\%) and also for eye movement disorders (14.4\%). Therefore, our study adds data to also support that the highest number of ocular conditions were present in the oldest stroke group. Regarding the neurological vision impairments associated with stroke because Rowe et al's cohort was studied prospectively,

Table 3 Number of Vision Impairment Codes per Patient by Age Group

\begin{tabular}{llllll}
\hline Vision Codes & $18-49$ & $50-64$ & $65-74$ & $75-84$ & $85+$ \\
\hline 1 & $377(12.6 \%)$ & $2263(11.5 \%)$ & $8237(11.5 \%)$ & $10,395(11.5 \%)$ & $10,844(12.8 \%)$ \\
2 & $88(2.9 \%)$ & $458(2.3 \%)$ & $1514(2.1 \%)$ & $1867(2.1 \%)$ & $2196(2.6 \%)$ \\
$3+$ & $31(1.0 \%)$ & $142(0.7 \%)$ & $456(0.6 \%)$ & $537(0.6 \%)$ & $496(0.6 \%)$ \\
\hline
\end{tabular}


Table 4 Vision Codes by Elixhauser Comorbidity

\begin{tabular}{|c|c|c|c|c|c|c|c|c|}
\hline Comorbidity & $\mathrm{N}$ & $\begin{array}{l}\text { Any Vision } \\
\text { Code }\end{array}$ & $\begin{array}{l}\text { Most Common } \\
\text { Code }\end{array}$ & N (\%) & $\begin{array}{l}\text { Second Most } \\
\text { Common Code }\end{array}$ & N (\%) & $\begin{array}{l}\text { Third Most } \\
\text { Common Code }\end{array}$ & $N(\%)$ \\
\hline Chronic Heart Failure & 37,627 & 5969 (15.9\%) & $\mathrm{H} 409$ & $\begin{array}{l}1273 \\
(3.4 \%)\end{array}$ & H3530 & $812(2.2 \%)$ & $\mathrm{H} 532$ & $462(1.2 \%)$ \\
\hline Valvular Disease & 13,807 & $2950(21.4 \%)$ & $\mathrm{H} 409$ & $657(4.8 \%)$ & H3530 & 469 (3.4\%) & H532 & $298(2.2 \%)$ \\
\hline Pulmonary Circulation Disease & 1798 & $279(15.5 \%)$ & $\mathrm{H} 409$ & $67(3.7 \%)$ & H3530 & $24(1.3 \%)$ & H53462 & $18(1.0 \%)$ \\
\hline Peripheral Vascular Disease & 18,203 & 3417 (18.8\%) & $\mathrm{H} 409$ & $690(3.8 \%)$ & H3530 & $440(2.4 \%)$ & H532 & $307(1.7 \%)$ \\
\hline Hypertension & $\begin{array}{l}207 \\
517\end{array}$ & $\begin{array}{l}34,413 \\
(16.6 \%)\end{array}$ & H409 & $\begin{array}{l}7399 \\
(3.6 \%)\end{array}$ & $\mathrm{H} 3530$ & $\begin{array}{l}4106 \\
(2.0 \%)\end{array}$ & H532 & $\begin{array}{l}3228 \\
(1.6 \%)\end{array}$ \\
\hline Paralysis & 22,930 & $3277(14.3 \%)$ & H409 & $642(2.8 \%)$ & H3530 & $343(1.5 \%)$ & H532 & $289(1.3 \%)$ \\
\hline Other Neurological Disorders & 14,685 & $1567(10.7 \%)$ & $\mathrm{H} 409$ & $401(2.7 \%)$ & H3530 & $225(1.5 \%)$ & H548 & $94(0.6 \%)$ \\
\hline Chronic Pulmonary Disease & 39,337 & $6115(15.5 \%)$ & H409 & $\begin{array}{l}1199 \\
(3.0 \%)\end{array}$ & H3530 & $803(2.0 \%)$ & H532 & $576(1.5 \%)$ \\
\hline $\begin{array}{l}\text { Diabetes without Chronic } \\
\text { Complications }\end{array}$ & 53,231 & 7227 (13.6\%) & H409 & $\begin{array}{l}1648 \\
(3.1 \%)\end{array}$ & H3530 & $721(1.4 \%)$ & H532 & $700(1.3 \%)$ \\
\hline $\begin{array}{l}\text { Diabetes with Chronic } \\
\text { Complications }\end{array}$ & 37,541 & 8054 (21.5\%) & E11319 & $\begin{array}{l}1628 \\
(4.3 \%)\end{array}$ & H409 & $\begin{array}{l}1304 \\
(3.5 \%)\end{array}$ & H532 & 767 (2.0\%) \\
\hline Hypothyroidism & 37,092 & 7022 (18.9\%) & $\mathrm{H} 409$ & $\begin{array}{l}1742 \\
(4.7 \%)\end{array}$ & H3530 & $\begin{array}{l}1094 \\
(2.9 \%)\end{array}$ & H532 & $541(1.5 \%)$ \\
\hline Renal Failure & 41,135 & 7504 (18.2\%) & $\mathrm{H} 409$ & $\begin{array}{l}1501 \\
(3.6 \%)\end{array}$ & E11319 & 817 (2.0\%) & H3530 & 785 (1.9\%) \\
\hline Liver Disease & 2866 & $453(15.8 \%)$ & $\mathrm{H} 409$ & $78(2.7 \%)$ & H532 & $56(2.0 \%)$ & H3530 & $35(1.2 \%)$ \\
\hline Peptic Ulcer Disease & 1679 & $332(19.8 \%)$ & H409 & $76(4.5 \%)$ & H3530 & $37(2.2 \%)$ & H532 & $32(1.9 \%)$ \\
\hline $\begin{array}{l}\text { Acquired Immunodeficiency } \\
\text { Syndrome }\end{array}$ & 400 & $44(11.0 \%)$ & H409 & $13(3.3 \%)$ & * & * & $*$ & * \\
\hline Lymphoma & 1369 & $239(17.5 \%)$ & $\mathrm{H} 409$ & $38(2.8 \%)$ & H532 & $30(2.2 \%)$ & H3530 & $21(1.5 \%)$ \\
\hline Metastatic Cancer & 2268 & $310(13.7 \%)$ & $\mathrm{H} 409$ & $50(2.2 \%)$ & H3530 & $31(1.4 \%)$ & H538 & $28(1.2 \%)$ \\
\hline Solid Tumor without Metastasis & 6626 & $955(14.4 \%)$ & $\mathrm{H} 409$ & $231(3.5 \%)$ & H3530 & $122(1.8 \%)$ & H538 & $65(1.0 \%)$ \\
\hline Rheumatoid Arthritis & 7296 & $1299(17.8 \%)$ & $\mathrm{H} 409$ & $287(3.9 \%)$ & H3530 & $153(2.1 \%)$ & H532 & $112(1.5 \%)$ \\
\hline Coagulopathy & 5790 & $1150(19.9 \%)$ & $\mathrm{H} 409$ & $225(3.9 \%)$ & H3530 & $139(2.4 \%)$ & H532 & $116(2.0 \%)$ \\
\hline Obesity & 18,427 & $3616(19.6 \%)$ & $\mathrm{H} 409$ & $555(3.0 \%)$ & H532 & $474(2.6 \%)$ & E11319 & $350(1.9 \%)$ \\
\hline Weight Loss & 7586 & 1309 (17.3\%) & H409 & $294(3.9 \%)$ & H3530 & $153(2.0 \%)$ & H532 & $84(1.1 \%)$ \\
\hline Fluid/Electrolyte Disorders & 28,909 & $5929(20.5 \%)$ & H409 & $\begin{array}{l}1249 \\
(4.3 \%)\end{array}$ & H3530 & $676(2.3 \%)$ & H532 & $577(2.0 \%)$ \\
\hline Chronic Blood Loss Anemia & 808 & $133(16.5 \%)$ & $\mathrm{H} 409$ & $37(4.6 \%)$ & H3530 & $17(2.1 \%)$ & H532 & $12(1.5 \%)$ \\
\hline Deficiency Anemias & 33,282 & $6290(18.9 \%)$ & $\mathrm{H} 409$ & $\begin{array}{l}1476 \\
(4.4 \%)\end{array}$ & H3530 & $713(2.1 \%)$ & H532 & $447(1.3 \%)$ \\
\hline Alcohol Abuse & 5570 & 869 (15.6\%) & H532 & $122(2.2 \%)$ & $\mathrm{H} 409$ & $112(2.0 \%)$ & H538 & 75 (1.3\%) \\
\hline Drug Abuse & 1621 & $229(14.1 \%)$ & H532 & $30(1.9 \%)$ & $\mathrm{H} 409$ & $25(1.5 \%)$ & H53462 & $20(1.2 \%)$ \\
\hline Psychoses & 6744 & 1014 (15.0\%) & $\mathrm{H} 409$ & $210(3.1 \%)$ & H3530 & $90(1.3 \%)$ & H532 & $79(1.2 \%)$ \\
\hline Depression & 44,988 & 8093 (18.0\%) & H409 & $\begin{array}{l}1724 \\
(3.8 \%)\end{array}$ & H3530 & 992 (2.2\%) & H532 & $726(1.6 \%)$ \\
\hline
\end{tabular}

Notes: H409- unspecified glaucoma; E11319- type 2 diabetes mellitus with unspecified diabetic retinopathy without macular edema; H532- diplopia; H3530unspecified macular degeneration; H53462- homonymous bilateral field defects, left side; H538- other visual disturbances; H548- legal blindness, as defined in United States of America

the assessment battery was intentional and comprehensive, and the researchers had access to the persons' ocular history within the year prior to stroke. Therefore, different neurological codes were identified such as: visual field loss, ocular motility problems, visual perception disorders, as compared to our study. The only the similar codes which could be a result of the stroke were diplopia (ocular motility problem) and visual field deficits [3].

Several limitations may affect the interpretation of our findings. First, this study was conducted among Medicare beneficiaries in the U.S. and may not be generalizable to 
stroke survivors not covered by Medicare or in settings outside of the U.S. Second, data were collected primarily for billing and administrative purposes. Certain codes may have been used more often than others since billing codes are linked to payment [17].Third, ocular diagnoses and current procedural terminology codes are usually found in the Medicare outpatient file (also known as the Carrier file), which is associated with institution care and ancillary care. We did not have the Carrier file, which requires submitting a finder file to the Research Data Assistance Center. Fourth, random and systematic coding errors can lead to under- or over-reporting of diagnoses. We were not able to determine if this data had errors since this is a retrospective review and we could not compare the claims data to electronic health records or clinical exam data. Fifth, we recognize that also because this is a retrospective review of cross sectional data, the ability to understand why there was lack of specificity, how the sequence of coding occurred, and information about the underutilization in certain codes is unknown. Also, we are not able to determine the type of visual assessments (e.g. ocular alignment and motility tests), used to determine the vision sequalae found among these stroke survivors, because this information is not listed in the claims files. Finally, as mentioned, a lack of vision screening or attention to vision in the post-acute setting may have resulted in underrepresentation of vision codes [14].

\section{Conclusions}

This study concludes that there is ocular comorbidity in a national stroke population. Our findings suggest that the full spectrum of ICD-10 codes for characterizing eye and vision disorders may be underutilized and lack specificity in the acute stroke population. Future studies might attempt to compare ophthalmic examination results with billing codes in order to characterize the type and frequency of ocular comorbidity among stroke survivors and the sensitivity and specificity of administrative claims data for identifying them. Additionally, future work should be done to classify which vision codes commonly applied in the acute stroke setting can be translated into actionable data. Comparison to a control group without stroke in future studies may also provide important insights into factors associated with variation in the application of eye and vision codes. In addition, it would be interesting to collect data on the pathogenesis (what proportion of ischemic stroke had central retinal occlusion or branch occlusion [18]) and investigate further how the use of ICD-10 codes impacts clinical decision making, recovery, and outcomes. Through tailored interventions, the specificity of coding with ICD-10 may encourage targeted strategies to manage, prevent, or delay the burden of ocular multimorbidity. Therefore, comprehensive screening of stroke related visual impairment should occur. We suggest it is essential and relevant that hospital providers pay attention to coding practices for ocular disorders among stroke survivors in order to facilitate referrals, appropriate interventions, and optimal visual outcomes.

\section{Abbreviations \\ ICD-10: Medicare International Statistical Classification of Diseases and Related Health Problems; U.S.: United States; PAC: Post Acute Care; HHA: Home Health Agency; SNF: Skilled Nursing Facility; IRF: Inpatient Rehabilitation Facility; LTAC: Long Term Assistive Care; ESRD: End Stage Renal Disease}

\section{Authors' contributions}

All authors have read and approved the manuscript. KPH was involved in the idea and development of manuscript as well as all aspects of the manuscript writing. SRF, TAR, KO, C-YL, JRE, DBW were all involve with the writing of this manuscript. HEW was involved in concept development, writing and mentoring $\mathrm{KPH}$. $\mathrm{AH}$ was responsible for the analysis.

\section{Funding}

Pepper Center Early Career Exchange Program Award, which is part of the Pepper Center Grant (P30AG024832), R01 HS024711 (PI: Reistetter); K12 HD0055929 (PI: Ottenbacher); K23EY027848 (PI: Ehrlich); K01HD101589 (PI: Li). This paper's contents are solely the responsibility of the authors and do not necessarily represent the official views of the NIH, NIA, or NEI. This funding supported the authors time to write this manuscript. The R01 award supported the use of the datasets.

\section{Availability of data and materials}

The data that support the findings of this study are available from Medicare but restrictions apply to the availability of these data, which were used under license for the current study, and so are not publicly available.

Ethics approval and consent to participate

Our ethics committee, at the University of Texas Medical Branch, ruled that no formal ethics approval was required in this particular research project. Additionally, this study used de-identified data, thus there was no consent involved. The Institutional Review Board at the University of Texas Medical Branch approved this study, which included the waiver to not need consent. A Data Use Agreement was completed following Centers for Medicare \& Medicaid Services requirements.

Consent for publication

Not applicable.

\section{Competing interests}

The authors declare that they have no competing interests.

\section{Author details}

'Division of Rehabilitation Sciences, University of Texas Medical Branch, School of Health Professions, 301 University Blvd., Galveston, TX 77555, USA. ${ }^{2}$ Physical Therapy Department, University of Texas Medical Branch, School of Health Professions, 301 University Blvd., Galveston, TX 77555, USA.

${ }^{3}$ Department of Occupational Therapy, School of Health Professions, University of Texas, 7703 Floyd Curl Drive, San Antonio, TX 78229, USA. ${ }^{4}$ Department of Preventive Medicine and Population Health, University of Texas Medical Branch, 301 University Blvd., Galveston, TX 77555, USA.

${ }^{5}$ Occupational Therapy Department, University of Texas Medical Branch, School of Health Professions, 301 University Blvd., Galveston, TX 77555, USA.

${ }^{6}$ Department of Ophthalmology and Visual Sciences, Kellogg Eye Center, University of Michigan, 100 Wall Street, Ann Arbor, Ml 48105, USA.

${ }^{7}$ Department of Ophthalmology, Duke University School of Medicine, 2351 Erwin Rd, Durham, NC 27705, USA. ${ }^{8}$ Department of Medicine, Duke University School of Medicine, Durham, NC, USA. ${ }^{9}$ Center for the Study of Aging and Human Disease, Duke University School of Medicine, Durham, NC, USA. ${ }^{10}$ Geriatrics Research Education and Clinical Center, Durham VA Medical Center, 8 Searle Center Drive, Durham, NC 27710, USA. 
Received: 14 April 2020 Accepted: 29 June 2020

Published online: 08 July 2020

\section{References}

1. Roger VL, et al. Heart disease and stroke statistics--2011 update: a report from the American Heart Association. Circulation. 2011;123:e18-e209.

2. Hepworth $L$, et al. Post-stroke visual impairment: a systematic literature review of types and recovery of visual conditions. Ophthalmol Res Int J. 2016:5:1-43.

3. Rowe FJ, et al. High incidence and prevalence of visual problems after acute stroke: an epidemiology study with implications for service delivery. PLoS One. 2019;14:e0213035.

4. Rowe FJ. Vision in stroke cohort: profile overview of visual impairment. Brain Behav. 2017;7.

5. Smith TM, Pappadis MR, Krishnan S, Reistetter TA. Stroke survivor and caregiver perspectives on post-stroke visual concerns and long-term consequences. Behav Neurol. 2018;2018(1463429).

6. Cheung N, Mitchell P, Wong TY. Diabetic retinopathy. Lancet. 2010; 376:124-36.

7. Chou R, Dana T, Bougatsos C, Grusing S, Blazina I. Screening for impaired visual acuity in older adults: updated evidence report and systematic review for the US preventive services task force. JAMA. 2016;315:915-33.

8. ICD - ICD-10-CM - International Classification of Diseases, Tenth Revision, Clinical Modification. https://www.cdc.gov/nchs/icd/icd10cm.htm (2019).

9. NQF: Risk Adjustment for Socioeconomic Status or Other Sociodemographic Factors - Description. http://www.qualityforum.org/ProjectDescription. aspx?project|D=73517.

10. Quan H, et al. Coding algorithms for defining comorbidities in ICD-9-CM and ICD-10 administrative data. Med Care. 2005;43:1130-9.

11. Sung S-F, et al. A comparison of stroke severity proxy measures for claims data research: a population-based cohort study. Pharmacoepidemiol Drug Saf. 2016;25:438-43.

12. Zheng DD, et al. Patterns of chronic conditions and their association with visual impairment and health care use. JAMA Ophthalmol. 2020. https://doi. org/10.1001/jamaophthalmol.2020.0052.

13. Umfress AC, Brantley MA. Eye care disparities and health related consequences in elderly patients with age-related eye disease. Semin Ophthalmol. 2016;31:432-8.

14. Rowe FJ, et al. Care provision for poststroke visual impairment. J Stroke Cerebrovasc Dis Off J Natl Stroke Assoc. 2015;24:1131-44

15. Rahmani B, et al. The cause-specific prevalence of visual impairment in an urban population. The Baltimore Eye Survey Ophthalmology. 1996; 103:1721-6.

16. Genetics of Glaucoma in People of African Descent (GGLAD) Consortium et al. Association of Genetic Variants With Primary Open-Angle Glaucoma Among Individuals With African Ancestry. JAMA 322, 1682-1691 (2019).

17. van Walraven $C$, Austin P. Administrative database research has unique characteristics that can risk biased results. J Clin Epidemiol. 2012;65:126-31.

18. French DD, Margo CE, Greenberg PB. Ischemic stroke risk in Medicare beneficiaries with central retinal artery occlusion: a retrospective cohort study. Ophthalmol Ther. 2018;7:125-31.

\section{Publisher's Note}

Springer Nature remains neutral with regard to jurisdictional claims in published maps and institutional affiliations.

Ready to submit your research? Choose BMC and benefit from:

- fast, convenient online submission

- thorough peer review by experienced researchers in your field

- rapid publication on acceptance

- support for research data, including large and complex data types

- gold Open Access which fosters wider collaboration and increased citations

- maximum visibility for your research: over $100 \mathrm{M}$ website views per year

At BMC, research is always in progress.

Learn more biomedcentral.com/submissions 